\title{
Engineering of Service Oriented Collaborative Network
}

\author{
Nesrine Elhabib, Xavier Boucher, and Sophie Peillon \\ Ecole des Mines de Saint Etienne \\ 158 cours fauriel, centre G2I \\ 42023 Saint Etienne, France \\ \{elhabib, boucher, peillon\} @emse.fr
}

\begin{abstract}
This paper presents an engineering approach of product/service oriented value creation chains. When configuring product/service productive networks, a high variability in alternative scenarios have to be considered due to flexibility levers introduced by the service oriented approach. To cope with the difficulty of scenario selection a 2 stages engineering approach is proposed. The first stage is developed in this paper, aiming at a general analysis of alternative scenarios: a network model and an evaluation system for scenario comparison are explained and discussed.
\end{abstract}

Keywords: Service oriented enterprise, Collaborative networks analysis, Collaboration scenario, Engineering methodology.

\section{Introduction}

In the paradigm of service-oriented enterprises, the dynamic configuration of valuecreation chains is addressed by a networking approach of Business Service (BS) supplied by several companies acting as service providers. In relation with the functionaleconomy model [1], we will consider that these value creation chains deliver integrated product-service systems (PSS) which mix tangible products with intangible services [2]. New enterprise engineering models are required to support the configuration of such a service-oriented network. Notably, the objective is to fully integrate the concept of service at the business level, and cope with the new degree of agility induced by the service approach [6]. This paper addresses this issue.

Several authors have already contributed to the engineering of service-oriented value-creation chains. Nicola Morelli [3] suggests an engineering method based on 3 levels: (1) mapping of the actors involved and of their interactions; (2) analysis of the logical and physical connections between the different stages and components of the system; (3) analysis of possible scenarios and uses. The main contribution of this work lies in showing how the components of the organizational system influence the PSS delivered. Tomohiko Sakao et al. [4] put forward a new "service-engineering" method, which takes into account the customer satisfaction and aims at designing sustainable product-services. The authors introduce the concept of receiver state parameters RSPs used to support the design of the PSS model, with their computer application («Service Explorer »). Nevertheless, they have not really address the design of the productive system which provides the PSS. 
The engineering of PSS value-creation chains should contain a comparative analysis of alternative PSS delivery scenarios. Several papers discuss indicators systems in this perspective. Jian-Hia Yang [5] proposes an approach based on balanced scorecard adapted to PSS delivery. While, Zeng Sen and al. [6] suggest an assessment methodology with several steps including simulation and optimization mechanisms. However, these papers are in line with a detailed engineering approach, and thus require very accurate data to correctly evaluate scenarios. More general works suggest an overall evaluation, such as MacDonald [7] who tries to assess the "value-in-use" of the product-service, or Omann [8] who assesses the sustainability of a PSS. But this kind of assessment only focuses on PSS supplied, without any real evaluation of the organizational delivery system.

Our paper presents an engineering method to construct Business Service Networks which meet specific customer needs. A conceptual framework is provided for this approach, and briefly illustrated by an "academic" case study. In section 2 an overview of the engineering method is given. Then, a structural model of service-oriented enterprise networks is specified. In section 4, an indicator system is discussed allowing the assessment of alternative organizational scenarios. Finally, the positioning of the approach and concluding remarks are provided.

\section{Overview of the Approach}

\subsection{An Engineering Method Based on Two Models}

The first objective of our approach is to manage, directly at the business level, the interoperability among the partners of the value creation chain. Consequently, the concept of "Business Service" is central to the method. The combination of BS generates a high number of alternative scenarios, induced by demand variability (e.g. distinct service level) or by solution flexibility (e.g. alternative providers). This engineering approach can provide a decision support to analyze and select suitable configurations (i.e. scenarios) of the PSS delivery system.

Another objective of our approach is to integrate the requirements of the "Functional Economy" Business Model [1]. This concept has been developed in economic sciences as a model of value creation for PSS, which integrates sustainable development objectives. Thus, as a base-hypothesis, we introduce sustainable development decision criteria into the engineering approach for the productive system. The system of indicators will be explained more deeply in section 4 .

Our network engineering approach proposed hereafter is composed of two principal stages: both a structural and dynamic analysis, each of which requires a distinct model to further develop decision aids.

- The structural or static analysis of the network: defines a structural mapping of the organizational network, corresponding to a scenario of customer needs satisfaction. Several alternative solutions and their corresponding networks are possible, and the model compares the different networks, through a strategic analysis of organizational configurations, corresponding to a step of general design of the network. 
- The dynamic composition for Business Service (BS) is based on the modeling and analysis of BS interconnectivity, flows exchanges and a temporal chain of events. This dynamic model can analyze the dynamic performances of the service delivery processes, taking into account working parameters, constraints and uncertainty along the PSS life-cycle. This model requires much more accurate information on the BS Network at the design stage.

Next, the structural model of the productive network will be presented.

\subsection{Case Study}

The service-oriented approach can manage and compare various organizational scenarios induced by the high uncertainty on the client behavior but also due to the flexibility of networks according to the principles of Business Services composition. To illustrate this notion of scenario we consider an example of a vehicle repair service (with a single damaged part). We deal with three typical scenarios, with different service levels and customer participation:

- Scenario 1: the damaged part is completely replaced by an identical piece by the garage. This scenario includes the purchase of a substitute part and the disposal of damaged part. The customer can borrow a vehicle until the repair is effectuated.

- Scenario 2: the damaged piece is repaired by the garage. Again, the customer can borrow a vehicle for the duration.

- Scenario 3: garage facilities are provided to customers, where they will repair their vehicle. Replacement parts can be bought and diverse tools rented.

These 3 generic scenarios can lead to many 'sub-scenarios', related to various business services offerings. The structural and static analysis model and dynamic composition of SM are intended to provide decision support for analyzing and selecting scenarios.

\section{Static and Structural Model to Analyze Productive Network Scenarios}

The static and structural analysis ( $1^{\text {st }}$ step of the engineering approach) provides a comparative assessment of different scenarios, as part of a general design phase. A structural model of the network (Figure 1 and Annex) is proposed, to organize all relevant information for the subsequent assessment procedure (developed in 4). Each scenario corresponds to a network of organizations of business services (BS). Each BS encapsulates business activities and competencies. This model (Figure 1) is based on 3 key principles:

1. Organizational entities of the production system. To meet the needs of structural analysis, the network is represented by 3 organizational entities: a "Business services", "Interface" between BS, and «Infrastructure components". In a service approach, Infrastructure acts supports production processes performed by business services. 
2. Considering the life cycle. Consistent with the service approach, we position the organizational entities within the life cycle of a PSS. Business services of each scenario are distributed throughout the full life cycle. Each scenario can be differentiated by covering more or less stages of the life cycle.

3. Three distinct functional layers. In reference to the separation of Back and Front Office plus Infrastructure, we consider a production oriented service system consisting of these 3 distinct functional layers [9], [10] that mobilize different competencies: a "User management" function for managing customer relationships through a "service agreement" process, a "PSS providing" function that includes the production process of PSS and the "Infrastructure" function which supports to the other functions.

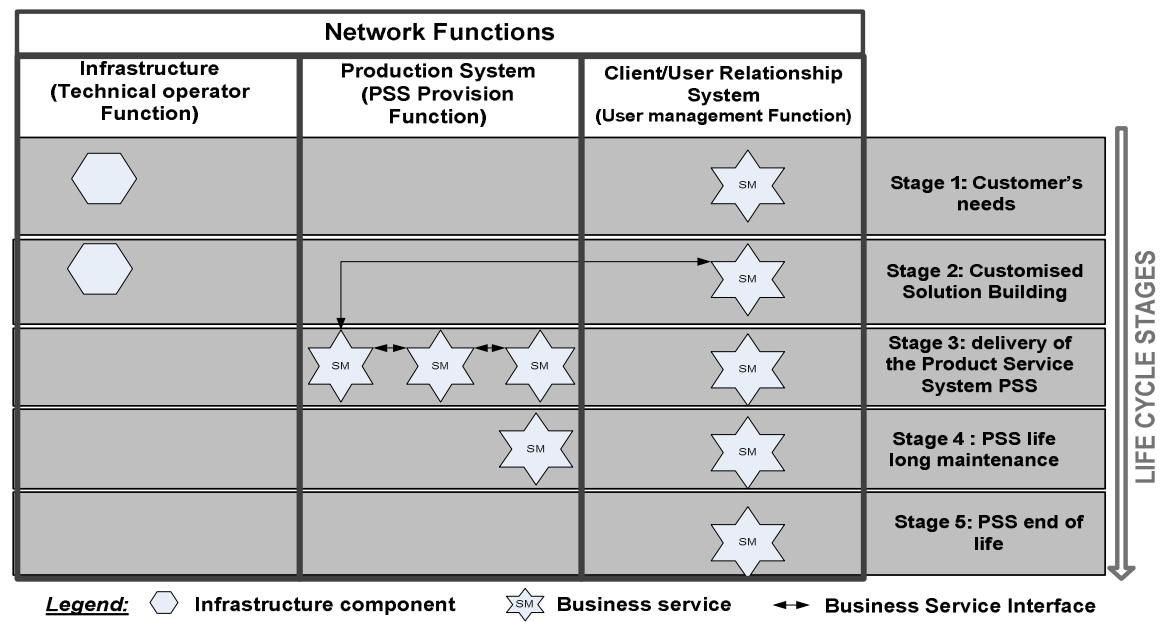

Fig. 1. Static and structural Model of the productive network

This structural model is associated with a logic process diagram providing a general description of the PSS production steps. The diagram consists of a series of activities or sub processes. The main process that ensures the expected PSS delivery can be associated to secondary processes indirectly involved (e.g., substitution part manufacturing in scenario 1 of the example) which contribute to the adaptability to the different levels of service required. The application of the model to this case study is presented in the annex.

\section{Comparative Analysis of the SPOS}

\subsection{Definitions Points of Views for Assessment as Well as Generic Indicators}

To specify the evaluation system, we start by translating the evaluation objectives into various assessment viewpoints, represented by the 3 axes in Figure X. The definition of these perspectives follows 3 principles:

- Necessity to take into account the different aspects of sustainable development: economic, societal and environmental assessments. 
- Represent the actors' intentions. In our context, there are two main stakeholders are involved in service delivery: the productive network and the customer. Moreover, since our approach lies in the deployment of sustainable development, we propose to consider a third actor the "Territorial actor"1.

- Opportunities and risks are evaluated at a rather strategic level of engineering of the production system via standard methods of strategic analysis as the SWOT method.

These principles generate 3 evaluation axis (axes "Sustainable development", "intentionality", "strategic factor") defining the generic evaluation structure of Figure 2.

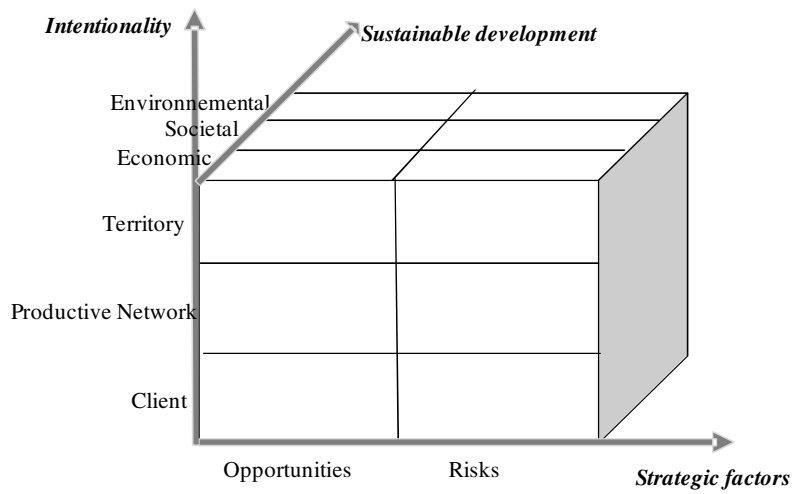

Fig. 2. Generic Evaluation Structure

This evaluation framework is very broad, and according to the specific case considered, all views are not systematically necessary. This framework is therefore a generic grid that guides the construction of indicators but should be adapted to any specific network considered. During this transition from the generic to the particular, we systematically maintain all facets of "Intentionality" and "Strategic factors" axes. However, the 3 facets of the axes "Sustainable Development" are not necessarily represented, as illustrated below for the case study presented in Section 3.

The analysis of the case study, only define 6 generic indicators specified in Table1:

- For the case study, the environmental dimension for the "Territorial" actor is illustrated by "Territorial risks" assessment. Given the strategic level of the analysis, environmental impact is only assessed at the level of the territory, and not analyzed more in detail for the other stakeholders. However, the application of the evaluation factor analysis specified in 4.2 will identify detailed risk factors contributing to the overall "Territorial risks".

- The societal dimension is represented through the points of the client standpoint (with the indicators "Potential of value creation" and "Risk of inadapted answer") and of the territorial actor (Indicator "Potential of territorial value creation"). In this case, there is a convergence of the societal and economic evaluations: the 2 indicators of

${ }^{1}$ The 'territory 'represents the territorial environment of the service oriented enterprise network, including economic, socio-human and ecological dimensions. 
value creation simultaneously evaluate a societal preoccupation and an economic dimension. There is little need to separate the 2 evaluations in this example.

Since this evaluation a aforementioned is situated at a strategic analysis level. The economic dimension is formalised by value creation indicators, and not by costoriented evaluation. In fact, a cost evaluation would require a more detailed analysis of the productive processes which will be developed in the 2 nd step of the engineering approach (dynamic evaluation).

Table 1. Generic indicators for the case study

\begin{tabular}{|l|l|l|l|}
\hline & «Territorial» Actor & Actor « network» & Actor « client » \\
\hline Opportunities & Territorial value creation & $\begin{array}{l}\text { Value creation for the } \\
\text { network }\end{array}$ & $\begin{array}{l}\text { Value creation for the } \\
\text { client }\end{array}$ \\
\hline Risks & Territorial risks & $\begin{array}{l}\text { Organisational risks for } \\
\text { the network }\end{array}$ & $\begin{array}{l}\text { Risks of inadapted } \\
\text { answer }\end{array}$ \\
\hline
\end{tabular}

After having defined the dimensions and generic indicators of the evaluation systems, a qualitative analysis of evaluation factors is developed in the following section.

\subsection{Qualitative Analysis of Evaluation Factors}

This analysis will detail and formalise the generic indicators. 3 key concepts are used to build the qualitative description of the evaluation factors (fig. 3):

- Aggregated indicators: represent the risks and opportunities for the 3 actors of the intentionality axis. 2 aggregated indicator levels are distinguished: the generic indicators identified in table 1 are evaluated by aggregation of more contextualized indicators depending on each case study.

- Explicative factors represent all the pertinent factors to be considered for the evaluation of aggregated indicators. This factors can be linked one with another by a qualitative link of "influence relationship". They can be associated to qualitative or quantitative metrics.

- Performance drivers: they represent the concrete levers of the service-oriented enterprise network which have an influence on the explicative factors and, consequently, on the aggregated indicators. In this approach, the drivers are the structural components (Business services, BS Interfaces, Infrastructure components) of the 3 organisational levels of the service oriented network.

Based on these key concepts, the qualitative analysis can build qualitative diagrams of influence relationships among performance drivers, explicative factors and aggregated indicators. These influence diagrams are necessary to cope with the complexity of performance factors considered, before further formalising a quantitative metric. For the vehicle repair case study, the 6 indicators of table 1 have to be analysed. Figure 3 shows an example with the generic indicator "Territorial value creation".

Figure 3 demonstrates that the generic indicator is first devided into 3 contextualized indicators which formalized the vision of the territorial actor : «Employment development », «Development of territorial competences », «Development of territorial attractivity ». The contextualized indicators are further elaborated by a network of explicative 
factors, linked to drivers constituted by the structural components of the enterprise network. These structural components are described in the model of section 3. For each scenario, the characteristics of the Business Services, BS interfaces and infrastructure components will have an impact of the explicative factors, making possible a comparative analysis of the scenarios.

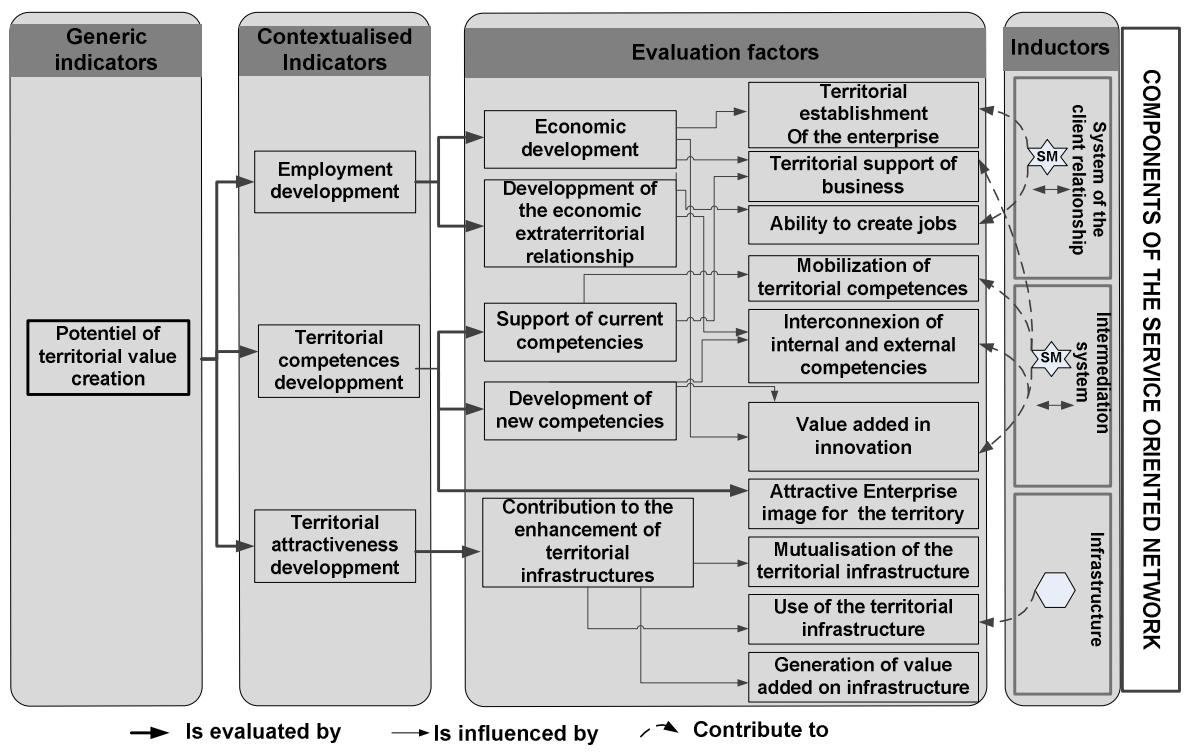

Fig. 3. Example of qualitative diagram of evaluation factors

\section{Positioning of This Approach and Conclusion}

Among the existing work we have targeted three specific approaches to the modeling of service-oriented production systems. Y. Shimomura [11] proposes a study of service-oriented manufacturing process based on state variables to represent the user. Nicolas Maussang [12] presented a PSS design method where models of service delivery scenarios are integrated within the design processes and tools. Third, Balin [13] has suggested a process-oriented simulation method for optimizing the quality of service-oriented production systems. Although complementary and using different formalisms (state agent models, design methodology, production system simulation), it should be noted that all these articles focus on the sequence of dynamic activities necessary to deliver services, in order to evaluate the quality of service. The approach developed here is complementary as we do not represent the dynamic process, but we only seek to represent the organizational structure of the service-oriented production systems: the distribution of actors and services across all layers and the functional interactions among these actors. Thus, in this first stage of general design of the production system, we aim to assess the relative contribution of alternative solutions to the organizational value creation expected by the client. This is an assessment of the 
quality of the structure organizational set up and not directly on the quality of service received.

Our next step will consist in building the quantitative metrics for comparative evaluation of scenarios. To answer the need of structural and static analysis of the networks, the optimisation or dynamic simulation methods generally used to evaluate service oriented systems (e.g [6], [12], [13]) are not relevant. For this static evaluation, we are going to use two complementary formalisms: the Fuzzy sub-set theory (to take in charge the coupling among qualitative and quantitative factors) and a multicriteria decision aid approach (to deal with the aggregation issue and the actor's points of view).

Acknowledgement. The authors wish to thank Chris Yukna for his help in English.

\section{References}

1. Stahel, W.: The Functional Economy: Cultural and Organizational Change. In: Richard, D.J. (ed.) The Industrial Green Game: Implications for Environmental Design and Management, pp. 91-100. National Academy Press, Washington (1997)

2. Michelini, R.C., Razzoli, R.P.: Product-service for environmental safeguard: a metrics to sustainability. Resources, Conservation and Recycling 42(1), 83-98 (2004)

3. Morelli, N.: Developping new product service system (PSS): methodologies and operational tools. Journal of cleaner production, Denmark (2006)

4. Tomohiko, S., Yoshiki, S.: Service Engineering: a novel engineering discipline for producers to increase value combining service and product. Journal of cleaner production, Japan (2006)

5. Yang, J.-H.: A balanced performance measurement scorecard approach for product service system. In: International Conference on Business Intelligence and Financial Engineering, China (2009)

6. Zeng, S., et al.: Service-oriented Business Process Modeling and Performance Evaluation based on AHP and Simulation. In: IEEE International Conference on e-Business Engineering (2007)

7. MacDonald, E.K., Martinez, V., Wilson, H.: Towards the Assessment of the Value-in-Use of Product-Service Systems: a Review. In: Performance Management Association Conference, New Zealand (2009)

8. Omann, I.: A Multicriteria tool for Evaluating the Impacts of Product Service Systems on Sustainable Development: An application in Austrian companies. Sustainable Europe Research Institute, SERI Working Papers, n5 (2007)

9. Tannery, F.: Internal report of the regional project SPOS. ENSMSE, France (October 2009)

10. Zarifian, P., Gadrey, J.: l'émergence d'un modèle de service: enjeux et réalités (2002)

11. Shimomura, Y., et al.: Service engineering-Methods and Tools for Effective PSS Development. In: Introduction to Product/Service-system Design. Springer, Heidelberg (2009)

12. Maussang, N.: Méthodologie de conception pour les systèmes produits-services, Institut polytechnique de Grenoble (2008)

13. Balin, S.: Amélioration de processus de production de services par la simulation, Université Paris Dauphine (2007) 